\title{
AN EFFECTIVE PAIR-POTENTIAL STUDY OF THE INTERACTION OF A WATER MONOMER WITH THE BASAL-PLANE SURFAGE OF ICE Ih
}

\author{
By B. N. Hale and J. Kiefer \\ (Graduate Center for Cloud Physics Research and Department of Physics, University of \\ Missouri-Rolla, Rolla, Missouri 654 or, U.S.A.)
}

\begin{abstract}
The central-force effective pair potential of Rahman and others (1975) is used to generate minimal binding-energy surfaces and configurations of a water monomer adsorbed on the unrelaxed basal plane surface of ice $\mathrm{Ih}$. In the initial study $\mathrm{H}_{2} \mathrm{O}$ molecules are placed with oxygen atoms fixed at the ice Ih lattice positions and associated hydrogens along the tetrahedral bond directions-restricting the $\mathrm{H}-\mathrm{O}-\mathrm{H}$ bond angles to $\mathrm{I} 09.5^{\circ}$. A rigid-body water monomer adsorbed on the surface interacts with all the $\mathrm{H}_{2} \mathrm{O}$ molecules in the bulk ice model via the central-force effective pair potential. The center-of-mass height, $z$, and the dipole orientation of the adsorbed $\mathrm{H}_{2} \mathrm{O}$ molecule are varied to minimize the total binding energy of the monomer over a particular $(x, y)$ point of the surface. Preliminary results showed that a rigid adsorbed monomer with an $\mathrm{H}-\mathrm{O}-\mathrm{H}$ angle fixed at $104.5^{\circ}$ produced only minor deviations in the binding-energy surfaces and that a large bulk-ice model (486 molecules) gave effectively the same results as a smaller ice model ( 50 molecules). Studies on an infinite ice surface using periodic boundary conditions are in progress and will be used to study surface relaxation and long-range effects on the minimal energy surfaces. Three types of sites on the basal plane were considered: (I) a site with all surface molecule protons pointing out of the surface; (2) a site with all surface molecule protons pointing into the surface; and (3) a site with all rows of surface molecule protons pointing alternately into and out of the surface. A "site" generally refers to a region (about $90 \AA^{2}$ ) on the surface over which the minimal binding-energy surface is to be determined.

Minimal binding-energy surfaces for the sites have been obtained together with plots showing the surface of the position of the center of mass as a function of $x$ and $y$. This is a model calculation and is limited by the validity of the potential under close scrutiny. However it gives a qualitative picture of how complex a terrain the real ice Ih surface might present to an adsorbed water molecule and how the water molecule might diffuse from site to site. From these calculations (and in the spirit of this model potential) a value for the mean path length and the mean residence time of the adsorbed water monomer can be estimated.
\end{abstract}

\section{REFERENGE}

Rahman, A., and others. 1975. Study of a central force model for liquid water by molecular dynamics, [by] A. Rahman, F. H. Stillingor and H. L. Lemberg. Journal of Chemical Physics, Vol. 63, No. 12, p. 5223-30. 